\title{
IDEAL PERTURBATION LEMMA
}

\author{
Martin Markl \\ Mathematical Institute of the Academy, Žitná 25 \\ 11567 Praha 1, The Czech Republic, \\ email: markl@math.cas.cz
}

\begin{abstract}
We explain the essence of perturbation problems. The key to understanding is the structure of chain homotopy equivalence - the standard one must be replaced by a finer notion which we call a strong chain homotopy equivalence.

We formulate an Ideal Perturbation Lemma and show how both new and classical (including the Basic Perturbation Lemma) results follow from this ideal statement.
\end{abstract}

\section{Introduction and results.}

All algebraic objects in this paper are defined over the ring of integers $\mathbb{Z}$. Our work was motivated by reconsidering the following classical Basic Perturbation Lemma (see [4] and the historical account there).

Recall that strong deformation retract $(S D R)$ data (also called a contraction) are given by chain complexes $\left(M, d_{M}\right),\left(N, d_{N}\right)$, chain maps $F:\left(M, d_{M}\right) \rightarrow\left(N, d_{N}\right)$, $G:\left(N, d_{N}\right) \rightarrow\left(M, d_{M}\right)$ and a chain homotopy $H: M \rightarrow M$ satisfying

$$
\begin{aligned}
F d_{M} & =d_{N} F, \\
G d_{N} & =d_{M} G, \\
G F-\mathbb{1}_{M} & =d_{M} H+H d_{M} \text { and } \\
F G & =\mathbb{1}_{N} .
\end{aligned}
$$

This of course means that $\left(N, d_{N}\right)$ is a strong deformation retract of $\left(M, d_{M}\right)$. One usually assumes that the following side conditions (also called annihilation properties) hold:

$$
H H=0, H G=0 \text { and } F H=0 .
$$

Then the following statement is true.

Basic Perturbation Lemma (BPL). Suppose we are given strong deformation data (⿴) satisfying (因) and a perturbation $\tilde{d}_{M}$ of the differential $d_{M}$ on $M$. Then

\footnotetext{
${ }^{1}$ Supported by the grant GA ČR 201/99/0675
} 
there are perturbations $\tilde{d}_{N}, \tilde{F}, \tilde{G}$ and $\tilde{H}$ of $d_{N}, F, G$ and $H$ that again form strong deformation data (四),

$$
\tilde{F} \tilde{d}_{M}=\tilde{d}_{N} \tilde{F}, \quad \tilde{G} \tilde{d}_{N}=\tilde{d}_{M} \tilde{G}, \quad \tilde{G} \tilde{F}-\mathbb{1}_{M}=\tilde{d}_{M} \tilde{H}+\tilde{H} \tilde{d}_{M} \quad \text { and } \tilde{F} \tilde{G}=\mathbb{1}_{N} .
$$

All notions used in the formulation of the BPL are standard and we believe it is not necessary to repeat their definitions here. Filtered objects and perturbations are treated in Section 3. The perturbation $\left(\tilde{d}_{N}, \tilde{F}, \tilde{G}, \tilde{H}\right)$ is given by the following explicit formulas (see again 顿):

$$
\begin{aligned}
\tilde{d}_{N} & =d_{N}+F\left(\partial_{M}+\partial_{M} H \partial_{M}+\partial_{M} H \partial_{M} H \partial_{M}+\partial_{M} H \partial_{M} H \partial_{M} H \partial_{M}+\cdots\right) G, \\
\tilde{F} & =F+F\left(\partial_{M}+\partial_{M} H \partial_{M}+\partial_{M} H \partial_{M} H \partial_{M}+\partial_{M} H \partial_{M} H \partial_{M} H \partial_{M}+\cdots\right) H, \\
\tilde{G} & =G+H\left(\partial_{M}+\partial_{M} H \partial_{M}+\partial_{M} H \partial_{M} H \partial_{M}+\partial_{M} H \partial_{M} H \partial_{M} H \partial_{M}+\cdots\right) G, \\
\tilde{H} & =H+H\left(\partial_{M}+\partial_{M} H \partial_{M}+\partial_{M} H \partial_{M} H \partial_{M}+\partial_{M} H \partial_{M} H \partial_{M} H \partial_{M}+\cdots\right) H,
\end{aligned}
$$

where $\partial_{M}:=\tilde{d}_{M}-d_{M}$. The formulas above contain infinite series, so one must assume some conditions assuring that they converge. This is usually achieved by assuming that both $\left(M, d_{M}\right)$ and $\left(N, d_{N}\right)$ are filtered complete, see again Section 3.

Our original motivation was to understand why there is such a formula and what is the role of side conditions. As usual, the best way to understand a problem is to formulate it in as general a form as possible. So let us consider the following:

Perturbation Problem (PP). Suppose we are given two complete filtered complexes $M=\left(N, d_{M}\right)$ and $N=\left(N, d_{N}\right)$ and chain maps $F: M \rightarrow N$ and $G: N \rightarrow M$ that are chain homotopy inverse to each other, with homotopies $H: M \rightarrow M$ and $L: N \rightarrow N$, that is

$$
F d_{M}=d_{N} F, G d_{N}=d_{M} G, G F-\mathbb{1}_{M}=d_{M} H+H d_{M}, F G-\mathbb{1}_{N}=d_{N} L+L d_{N} .
$$

Given a perturbation $\tilde{d}_{M}$ of the differential $d_{M}$, find perturbations $\tilde{d}_{N}, \tilde{F}, \tilde{G}, \tilde{H}$ and $\tilde{L}$ of $d_{N}, F, G, H$ and $L$ such that $\tilde{F}$ and $\tilde{G}$ are chain maps with respect to the perturbed differentials, homotopy inverse to each other, with homotopies $\tilde{H}$ and $\tilde{L}$, that is

$$
\tilde{F} \tilde{d}_{M}=\tilde{d}_{N} \tilde{F}, \tilde{G} \tilde{d}_{N}=\tilde{d}_{M} \tilde{G}, \tilde{G} \tilde{F}-\mathbb{1}_{M}=\tilde{d}_{M} \tilde{H}+\tilde{H} \tilde{d}_{M}, \tilde{F} \tilde{G}-\mathbb{1}_{N}=\tilde{d}_{N} \tilde{L}+\tilde{L} \tilde{d}_{N} .
$$

Observe that in the formulation of the BPL and the PP we consider not only the differentials and the chain maps, but also the homotopies to be a part of the structure which has to be perturbed. Ignoring homotopies leads to the 'crude' perturbation lemma formulated at the end of this Introduction.

The fact, both frustrating and provoking, is that the PP has, for general input data, no solution! - a rigorous formulation and proof of this negative statement 
is provided by Theorem 16. The reason is that the chain homotopy equivalence $(F, G, H, L)$ of (3) is not a homotopy invariant concept and it must be replaced by a subtler notion of a strong (chain) homotopy equivalence:

Definition 1. A strong homotopy equivalence (SHE) consists of degree $2 m$ maps $F_{2 m}: M \rightarrow N, G_{2 m}: N \rightarrow M$ and degree $2 m+1$ 'homotopies' $H_{2 m+1}: M \rightarrow M$, $L_{2 m+1}: N \rightarrow N$, for all $m \geq 0$, such that

$$
\begin{aligned}
F_{0} d_{M} & =d_{N} F_{0}, & G_{0} d_{N} & =d_{M} G_{0}, \\
G_{0} F_{0}-\mathbb{1}_{M} & =d_{M} H_{1}+H_{1} d_{M}, & F_{0} G_{0}-\mathbb{1}_{N} & =d_{N} L_{0}+L_{0} d_{N}
\end{aligned}
$$

and that, for each $m \geq 1$,

$$
\begin{aligned}
d_{N} F_{2 m}-F_{2 m} d_{N} & =\sum_{0 \leq i<m}\left(F_{2 i} H_{2(m-i)-1}-L_{2(m-i)-1} F_{2 i}\right), \\
d_{M} H_{2 m+1}+H_{2 m+1} d_{M} & =\sum_{0 \leq j \leq m} G_{2 j} F_{2(m-j)}-\sum_{0 \leq j<m} H_{2 j+1} H_{2(m-j)-1}, \\
d_{M} G_{2 m}-G_{2 m} d_{N} & =\sum_{0 \leq i<m}\left(G_{2 i} L_{2(m-i)-1}-H_{2(m-i)-1} G_{2 i}\right), \\
d_{N} L_{2 m+1}+d_{N} L_{2 m+1} & =\sum_{0 \leq j \leq m} F_{2 j} G_{2(m-j)}-\sum_{0 \leq j<m} L_{2 j+1} L_{2(m-j)-1} .
\end{aligned}
$$

See 8.1 where we expanded the axioms above for some small $m$. To understand better the meaning of a SHE, we offer the following analogy.

A homotopy associative algebra is a chain complex $V=\left(V, d_{V}\right)$ with a homotopy associative multiplication $\mu: V^{\otimes 2} \rightarrow V$ :

$$
\mu(\mu \otimes \mathbb{1})-\mu(\mathbb{1} \otimes \mu) \sim 0 \text { modulo a chain homotopy } \nu: V^{\otimes 3} \rightarrow V \text {. }
$$

As argued in [5], a proper homotopy invariant version of this concept is that of a strongly homotopy associative algebra, which is a structure consisting of infinitely many multilinear operations $\left\{\mu_{n}: V^{\otimes n} \rightarrow V\right\}_{n \geq 2}$ such that the 'multiplication' $\mu_{2}: V^{\otimes 2} \rightarrow V$ is homotopy associative up to the homotopy $\mu_{3}: V^{\otimes 3} \rightarrow V$, and there is, for each $n \geq 4$, a certain 'coherence relation' assumed to be zero modulo the homotopy $\mu_{n}$, see [6]. While each strongly homotopy associative algebra defines, by $\mu:=\mu_{2}$ and $\nu:=\mu_{3}$, a homotopy associative one, the converse is not true; there are obstructions for extending a homotopy associative multiplication to a strongly homotopy associative one.

The situation in Definition 1 is similar. While a strong homotopy equivalence defines, by $F:=F_{0}, G:=G_{0}, H:=H_{1}$ and $L:=L_{1}$ an ordinary homotopy equivalence, the converse is not true - there is a primary obstruction $[\mathfrak{o}]$ for extending a homotopy equivalence to a strong one. The surprising Theorem 11 says that vanishing of this primary obstruction already implies the existence of the extension. 
A strong homotopy equivalence of $M$ and $N$ will be denoted as $(\underline{F}, \underline{G}, \underline{H}, \underline{L}): M \rightarrow$ $N$. Let us formulate our Ideal Perturbation Lemma.

Ideal Perturbation Lemma (IPL). Suppose we are given two complete filtered complexes $M=\left(N, d_{M}\right)$ and $N=\left(N, d_{N}\right)$ and a strong homotopy equivalence $(\underline{F}, \underline{G}, \underline{H}, \underline{L}): M \rightarrow N$.

Given a perturbation $\tilde{d}_{M}$ of the differential $d_{M}$, there exist a perturbation $\tilde{d}_{N}$ of the differential $d_{N}$ and a perturbation $(\underline{\tilde{E}}, \underline{\tilde{G}}, \underline{\tilde{H}}, \underline{\tilde{L}})$ of $(\underline{F}, \underline{G}, \underline{H}, \underline{L})$ which is a strong homotopy equivalence of the perturbed complexes $\left(M, \tilde{d}_{M}\right)$ and $\left(N, \tilde{d}_{N}\right)$. Moreover, the perturbations $\tilde{d}_{M}$ and $(\underline{\tilde{F}}, \underline{\tilde{G}}, \underline{\tilde{H}}, \underline{\tilde{L}})$ depend functorially on $\tilde{d}_{M}$ and $(\underline{F}, \underline{G}, \underline{H}, \underline{L})$.

The IPL is proved in Section 6 , see also 8.6 for explicit formulas. As most ideal things, the Ideal Perturbation Lemma is almost useless. In practice, the input data are formulated only in terms of an ordinary homotopy equivalence, and the answer is also expected to be a perturbation of this ordinary homotopy equivalence. Here is our mundane version of the Ideal Perturbation Lemma.

Theorem 2. Suppose that the obstruction $[\mathfrak{o}]$ to the extension of the homotopy equivalence (3) to a strong one vanishes. Then the Perturbation Problem has a solution, functorial up to a choice of the extension of (3) to a strong homotopy equivalence.

The theorem immediately follows from the IPL and the above notes. There are situations when the obstruction $[\mathfrak{o}]$ vanishes and when there even exists a functorial extension of the homotopy equivalence (3) to a SHE. This the case of our motivating example of the Basic Perturbation Lemma (the case $L=0$ ). It immediately follows from Theorem 12 that the side conditions (2) guarantee the existence of a functorial extension of (1) to a strong homotopy equivalence. So Theorem 2 implies the BPL.

Another trick that overrides the nonexistence of a solution of the PP is to change the initial data a bit. We show in Theorem 13 that changing in (3) the homotopy $H$ to $H-G(F H-L F)$ (or, dually, $L$ to $L-F(G L-H G)$ ) annihilates the obstruction [o] and we reprove the following recent result by J. Huebschmann and T. Kadeishvili «1

Theorem 3. Let $M=\left(M, d_{M}\right)$ and $N=\left(N, d_{N}\right)$ be complete filtered chain complexes and $(F, G, H, L)$ a chain homotopy equivalence (3).

Given a perturbation $\tilde{d}_{M}$ of $d_{M}$, there exist a perturbation $\tilde{d}_{N}$ of the differential $d_{N}$ and a homotopy equivalence $(\tilde{F}, \tilde{G}, \tilde{H}, \tilde{L})$ of the perturbed complexes $\left(M, \tilde{d}_{M}\right)$ and $\left(N, \tilde{d}_{N}\right)$ that is a perturbation of $(F, G, H-G(F H-L F), L)$.

Changing $L$ to $L-F(G L-H G)$ and leaving $H$ untouched gives the following complement to Theorem 3 .

Complement to Theorem 3. Under the assumption of Theorem 6, there exists another perturbation $\tilde{d}_{N}^{\prime}$ of the differential $d_{N}$ and another homotopy equivalence 
$\left(\tilde{F}^{\prime}, \tilde{G}^{\prime}, \tilde{H}^{\prime}, \tilde{L}^{\prime}\right)$ of the perturbed complexes $\left(M, \tilde{d}_{M}\right)$ and $\left(N, \tilde{d}_{N}^{\prime}\right)$ that is a perturbation of $(F, G, H, L-F(G L-H G))$.

Ignoring the homotopies in the Perturbation Problem, we get the following

Crude Perturbation Lemma. Suppose we are given two complete filtered complexes $M=\left(N, d_{M}\right)$ and $N=\left(N, d_{N}\right)$ and chain maps $F: M \rightarrow N$ and $G: N \rightarrow M$ that are chain homotopy inverse to each other.

Given a perturbation $\tilde{d}_{M}$ of the differential $d_{M}$, there are perturbations $\tilde{d}_{N}, \tilde{F}$ and $\tilde{G}$ of $d_{N}, F$ and $G$ such that $\tilde{F}$ and $\tilde{G}$ are chain maps with respect to the perturbed differentials, homotopy inverse to each other.

A conceptual explanation of these results is given in Section 0 .

Plan of the paper: In Section 2 we recall colored operads and introduce the operad Iso describing isomorphisms of chain complexes. In Section 3 we repeat necessary facts on filtrations and perturbations and define the filtered operad $\mathcal{D}$ if describing perturbations of differentials. The filtered operad $\mathcal{R}_{\text {iso }}$ that describes strong homotopy equivalences is introduced in Section 1 where we also discuss extensions of a homotopy equivalence to a strong one. In Section 5 we introduce the operad $\tilde{\mathcal{R}}_{\text {iso }}$ for perturbations of strong homotopy equivalences and construct a retraction $r$ that gives the functorial solution to the IPL. Some of the proofs are postponed to Section 6. In Section 7 we give a conceptual explanation of the results. In the Appendix (Section 8) we present some explicit formulas.

\section{Language of operads.}

Roughly speaking, operads are objects that describe types of algebraic systems. Colored operads are then objects describing diagrams of algebraic systems. The definition of a (colored) operad is classical (see [1] or [5]) and we will not repeat it here in its full generality.

By an operad we will always mean an operad in the symmetric monoidal category Chain $\mathbb{Z}_{\mathbb{Z}}$ of differential graded complexes of abelian groups (that is, complexes of $\mathbb{Z}$ modules). Operads in this category behave in many aspects as associative algebras, so we may speak about suboperads, ideals, presentations, resolutions, etc., see [2].

All algebraic objects in this paper will have only unary operations. Colored operads describing algebraic systems with only unary operations are the same as small additive categories enriched over Chain $\mathrm{Z}_{\mathbb{Z}}$. This means that all hom-sets are chain complexes and composition maps are homomorphisms of chain complexes. All operads in this paper will be of this type.

We will use the 'operadic' notation and terminology. Thus, for such an op- 
$\operatorname{erad} /$ category $\mathcal{P}$, we call $\mathfrak{C}:=O b(\mathcal{P})$ the set of colors and, for $c, d \in \mathfrak{C}$, we denote

$$
\mathcal{P}\left(\begin{array}{l}
d \\
c
\end{array}\right):=\operatorname{Mor}_{\mathcal{P}}(c, d)
$$

We will usually express the fact that $f \in \mathcal{P}\left(\begin{array}{l}d \\ c\end{array}\right)$ by writing $f: c \rightarrow d$.

In the particular case when $\operatorname{card}(\mathfrak{C})=1$, the $\mathfrak{C}$-colored operads are exactly differential graded associative unital algebras. In this paper, by a colored operad we always mean an operad colored by the two-point set $\mathfrak{C}=\{\mathrm{B}, \mathrm{W}\}$ (B from black, $\mathrm{W}$ from white) or by a set isomorphic to this one.

Example 4. Let $M=\left(M, d_{M}\right)$ be a chain complex, then the endomorphism operad $\mathcal{E} n d_{M}$ is defined to be the chain complex $\operatorname{Hom}(M, M)$ with the operadic structure (which in this particular case is the same as that of an unital associative algebra) given by the composition. An algebra over an operad $\mathcal{P}$ is an operadic homomorphism $A: \mathcal{P} \rightarrow \mathcal{E} n d_{M}$. In this situation we also say that the operad $\mathcal{P}$ acts on the chain complex $M$.

Example 5. This example describes a colored version of the endomorphism operad recalled in Example 1 . Let $M=\left(M, d_{M}\right)$ and $N=\left(N, d_{N}\right)$ be chain complexes. By a colored endomorphism operad $\mathcal{E}_{n} d_{M, N}$ we mean the full subcategory of Chain $\mathrm{Z}_{\mathbb{Z}}$ with objects $M$ and $N$. If $\mathcal{P}$ is a $\{\mathrm{B}, \mathrm{W}\}$-colored operad, then by a $\mathcal{P}$-algebra we mean a homomorphism $A: \mathcal{P} \rightarrow \mathcal{E} n d_{M, N}$ such that $A(\mathrm{~B})=M$ and $A(\mathrm{~W})=N$.

Example 6. Let $f: \mathrm{B} \rightarrow \mathrm{W}, g: \mathrm{W} \rightarrow \mathrm{B}$ be two degree-zero generators and denote

$$
\mathcal{I} s o:=\left(\frac{\mathcal{F}(f, g)}{\left(f g=1_{\mathrm{W}}, g f=1_{\mathrm{B}}\right)}, d=0\right) \text {. }
$$

In the above display, $\mathcal{F}(f, g)$ denotes the free $\{\mathrm{B}, \mathrm{W}\}$-colored operad on the set $\{f, g\}$ and $\left(f g=1_{\mathrm{W}}, g f=1_{\mathrm{B}}\right)$ the operadic ideal generated by $f g-1_{\mathrm{W}}$ and $g f-1_{\mathrm{B}}$.

An algebra $A: \mathcal{I} s o \rightarrow \mathcal{E}_{n} d_{M, N}$ consists of two degree zero chain maps $F: M \rightarrow$ $N, G: N \rightarrow M$ that are inverse to each other. Thus the operad $\mathcal{I}$ so describes isomorphisms of chain complexes, whence its name.

\section{Filtrations and Perturbations.}

Let $M=\left(M, d_{M}\right)$ be a chain complex. A (descending) filtration on $M$ is a descending sequence $\left\{F^{p} M\right\}_{p \geq 0}$ of subcomplexes of $M$. If not stated otherwise, we always assume that the filtration is complete. This, by definition, means that the module $M$ is complete in the $F^{p}$-adic topology. This guarantees that each sum $\sum_{p \geq 0} m_{p}$ 
with $m_{p} \in F^{p} M$ represents a unique element of $M$. A typical example is the module of power series $\mathbb{Z}[[h]]$ with the filtration defined by $F^{p} \mathbb{Z}[[h]]:=h^{p} \mathbb{Z}[[h]], p \geq 0$.

Morphisms of filtered chain complexes are maps that preserve filtrations. A linear map $g: M \rightarrow N$ is a perturbation or deformation of a linear map $f: M \rightarrow N$ if

$$
(f-g)\left(F^{p} M\right) \subset F^{p+1} N \text { for each } p \geq 0 .
$$

If $M$ and $N$ are filtered complexes, then the chain complex $\operatorname{Hom}(M, N)$ is also filtered, by

$$
F^{q} \operatorname{Hom}(M, N):=\left\{f \in \operatorname{Hom}(M, N) ; f\left(F^{p} M\right) \subset F^{p+q} N \text { for each } p\right\} .
$$

We believe that the notion of a filtered algebra, operad, etc., is clear; we require that all structure operations preserve the filtration.

If $M=\left(M, d_{M}\right)$ is a filtered chain complex, then (4) defines a filtration of the endomorphism operad $\mathcal{E} n d_{M}$. A filtered algebra over a filtered operad $\mathcal{P}$ is a homomorphism $A: \mathcal{P} \rightarrow \mathcal{E} n d_{M}$ of filtered operads. There is an evident colored analog of this notion.

Example 7. Let $\bar{x}$ be a generator of degree -1 and let

$$
\text { preD if }:=(\mathcal{F}(\bar{x}), d) \text {, }
$$

with $d$ the 'derivation' in the operadic sense defined by $d \bar{x}:=-\bar{x} \bar{x}$. The free operad $\mathcal{F}(\bar{x})$ on $\bar{x}$ is the same as the polynomial ring $\mathbb{Z}[\bar{x}]$. We define the filtration

$$
F^{p} \text { pre } \mathcal{D} \text { if }:=\text { the subspace spanned by monomials in } \bar{x} \text { of length } \geq p \geq 0 .
$$

The differential $d$ clearly preserves the filtration, as well as does the composition, so the operad pre $\mathcal{D}$ if is filtered. Let $\mathcal{D}$ if be the completion of pre $\mathcal{D}$ if with respect to the above filtration; of course, $\mathcal{D}$ if coincides with the algebra of power series $\mathbb{Z}[[\bar{x}]]$.

Filtered $\mathcal{D}$ if-algebras $A: \mathcal{D}$ if $\rightarrow \mathcal{E} n d_{M}$ on $M=\left(M, d_{M}\right)$ correspond to perturbations $\tilde{d}_{M}=d_{M}+\partial_{M}$ of the differential $d_{M}$, the correspondence being given by $\partial_{M}:=A(\bar{x})$. Indeed, $d \bar{x}=-\bar{x} \bar{x}$ is mapped by $A$ to $\partial_{M} d_{M}+d_{M} \partial_{M}=-\partial_{M} \partial_{M}$, which is the same as $\left(d_{M}+\partial_{M}\right)^{2}=0$.

Proposition 8. The operad $\mathcal{D}$ if of Example $\mathbb{Y}$ is acyclic, that is, $H_{*}(\mathcal{D}$ if $) \cong \mathbf{1}$, where 1 is the trivial operad.

The proof of the above proposition is easy and we leave it as an exercise. One feels that the proposition must be 'philosophically' true. Algebras over the trivial operad $\mathbf{l}$ are just chain complexes with no additional structure, i.e. with only the structure given by the unperturbed differential. The operad $\mathcal{D}$ if describes perturbations of this differential, so it must be homologically the same as 1 . 


\section{Strong homotopy equivalences.}

In Example 6 we introduced a colored operad $\mathcal{I}$ so describing chain maps $F: M \rightarrow N$, $G: N \rightarrow M$ such that $F G=\mathbb{1}_{N}$ and $F G=\mathbb{1}_{M}$.

A general belief is that the homotopy analog of this situation is given by a quadruple $(F, G, H, L)$, where $F: M \rightarrow N$ and $G: N \rightarrow M$ are degree zero chain maps that are homotopy inverses of each other, with associated homotopies $H$ and $L$ :

$$
G F-\mathbb{1}_{M}=d_{M} H+H d_{M}, F G-\mathbb{1}_{N}=d_{N} L+L d_{N} .
$$

Such a quadruple is clearly an algebra over the operad

$$
\mathcal{R}_{\text {fake }}:=\left(\mathcal{F}\left(f_{0}, g_{0}, f_{1}, g_{1}\right), d\right),
$$

where

$$
f_{0}: \mathrm{B} \rightarrow \mathrm{W}, g_{0}: \mathrm{W} \rightarrow \mathrm{B}, f_{1}: \mathrm{B} \rightarrow \mathrm{B} \text { and } g_{1}: \mathrm{W} \rightarrow \mathrm{W}
$$

are generators with $\operatorname{deg}\left(f_{0}\right)=\operatorname{deg}\left(g_{0}\right)=0, \operatorname{deg}\left(f_{1}\right)=\operatorname{deg}\left(g_{1}\right)=1$, and the differential $d$ is given by

$$
d\left(f_{0}\right):=0, d\left(g_{0}\right):=0, d\left(f_{1}\right):=g_{0} f_{0}-1_{\mathrm{B}} \text { and } d\left(g_{1}\right):=f_{0} g_{0}-1_{\mathrm{W}} .
$$

There is a dg operad map $\alpha_{\text {fake }}: \mathcal{R}_{\text {fake }} \rightarrow \mathcal{I}$ so given by

$$
\alpha_{\text {fake }}\left(f_{0}\right):=f, \alpha_{\text {fake }}\left(g_{0}\right):=g, \alpha_{\text {fake }}\left(f_{1}\right):=0 \text { and } \alpha_{\text {fake }}\left(g_{1}\right):=0 \text {. }
$$

The following fact which shows that $\mathcal{R}_{\text {fake }}$ is not an acyclic resolution of the operad Iso is crucial.

Fact. The map $\alpha_{\text {fake }}$ is not a homology isomorphism. For instance, $f_{0} f_{1}-g_{1} g_{0}$ is a cycle in the kernel of $\alpha_{\text {fake }}$ that is not homologous to zero.

A proper resolution of $\mathcal{I}$ so was described in [5]. It is a graded colored differential operad

$$
\mathcal{R}_{\text {iso }}:=\left(\mathcal{F}\left(f_{0}, f_{1}, \ldots ; g_{0}, g_{1}, \ldots\right), d\right),
$$

with generators of two types,

$$
\begin{aligned}
& \text { (i) generators }\left\{f_{n}\right\}_{n \geq 0}, \operatorname{deg}\left(f_{n}\right)=n,\left\{\begin{array}{l}
f_{n}: \mathrm{B} \rightarrow \mathrm{W} \text { if } n \text { is even, } \\
f_{n}: \mathrm{B} \rightarrow \mathrm{B} \text { if } n \text { is odd, }
\end{array}\right. \\
& \text { (ii) generators }\left\{g_{n}\right\}_{n \geq 0}, \operatorname{deg}\left(g_{n}\right)=n,\left\{\begin{array}{l}
g_{n}: \mathrm{W} \rightarrow \mathrm{B} \text { if } n \text { is even, } \\
g_{n}: \mathrm{W} \rightarrow \mathrm{W} \text { if } n \text { is odd. }
\end{array}\right.
\end{aligned}
$$

The differential $d$ is given by

$$
\begin{aligned}
& d f_{0}:=0, \quad d g_{0}:=0, \\
& d f_{1}:=g_{0} f_{0}-1, \quad d g_{1}:=f_{0} g_{0}-1
\end{aligned}
$$


and, on remaining generators, by the formula

$$
\begin{aligned}
d f_{2 m} & :=\sum_{0 \leq i<m}\left(f_{2 i} f_{2(m-i)-1}-g_{2(m-i)-1} f_{2 i}\right), m \geq 0, \\
d f_{2 m+1} & :=\sum_{0 \leq j \leq m} g_{2 j} f_{2(m-j)}-\sum_{0 \leq j<m} f_{2 j+1} f_{2(m-j)-1}, m \geq 1, \\
d g_{2 m} & :=\sum_{0 \leq i<m}\left(g_{2 i} g_{2(m-i)-1}-f_{2(m-i)-1} g_{2 i}\right), m \geq 0, \\
d g_{2 m+1} & :=\sum_{0 \leq j \leq m} f_{2 j} g_{2(m-j)}-\sum_{0 \leq j<m} g_{2 j+1} g_{2(m-j)-1}, m \geq 1,
\end{aligned}
$$

see also (8.2). The above formulas can be written in a compact form by introducing elements

$$
\begin{array}{ll}
f_{\bullet}:=f_{0}+f_{2}+f_{4}+\cdots: \mathrm{B} \rightarrow \mathrm{W}, \quad h_{\bullet}:=f_{1}+f_{3}+f_{5}+\cdots: \mathrm{B} \rightarrow \mathrm{B}, \\
g_{\bullet}:=g_{0}+g_{2}+g_{4}+\cdots: \mathrm{W} \rightarrow \mathrm{B}, \quad l_{\bullet}:=l_{1}+l_{3}+l_{5}+\cdots: \mathrm{W} \rightarrow \mathrm{W} .
\end{array}
$$

Then $\mathcal{R}_{\text {iso }}=\mathcal{F}\left(f_{\bullet}, g_{\bullet}, h_{\bullet}, l_{\bullet}\right)$ with the differential given by

$d f_{\bullet}=f_{\bullet} h_{\bullet}-l_{\bullet} f_{\bullet}, d h_{\bullet}=g_{\bullet} f_{\bullet}-h_{\bullet} h_{\bullet}-1_{\mathrm{B}}, d g_{\bullet}=g_{\bullet} l_{\bullet}-h_{\bullet} g_{\bullet}$ and $d l_{\bullet}=f_{\bullet} g_{\bullet}-l_{\bullet} l_{\bullet}-1_{\mathrm{W}}$.

We will use this kind of abbreviation quite often, but we shall always keep in mind that each formula of this type in fact represents infinitely many formulas for homogeneous parts. The operad $\mathcal{R}_{\text {iso }}$ is 'trivially' filtered, by

$$
F^{p} \mathcal{R}_{\text {iso }}:= \begin{cases}\mathcal{R}_{\text {iso }}, & \text { for } p=0, \text { and } \\ 0, & \text { for } p>0 .\end{cases}
$$

This filtration is obviously complete. Algebras over the operad $\mathcal{R}_{\text {iso }}$ are the strong homotopy equivalences introduced in Definition 1 .

The following theorem, formulated without proof in [5], claims that $\mathcal{R}_{\text {iso }}$ is an acyclic resolution of the operad $\mathcal{I}$ so.

Theorem 9. The map $\alpha_{\text {iso }}: \mathcal{R}_{\text {iso }} \rightarrow \mathcal{I}$ so defined by

$$
\alpha_{\text {iso }}\left(f_{0}\right):=[f], \alpha_{\text {iso }}\left(g_{0}\right):=[g] \text {, while } \alpha_{\text {iso }}\left(f_{n}\right):=0, \alpha_{\text {iso }}\left(g_{n}\right)=0 \text { for } n \geq 1 \text {, }
$$

is a map of differential graded colored operads that induces an isomorphism of cohomology.

Proof. It is clear that $\alpha_{\text {iso }}$ commutes with the differentials and that it induces an isomorphism $H_{0}\left(\mathcal{R}_{\text {iso }}, d\right) \cong \mathcal{I}$ so. It thus remains to prove that $\mathcal{R}_{\text {iso }}$ is acyclic in positive dimensions.

The operad $\mathcal{F}\left(f_{0}, f_{1}, \ldots ; g_{0}, g_{1}, \ldots\right)$ is the free abelian group spanned by composable chains of generators. The length of these chains induces another grading, which 
we call the homogeneity. The differential $d$ decomposes as $d=d_{-1}+d_{+1}$, where $d_{i}$ raises the homogeneity by $i= \pm 1$. Explicitly, $d_{+1}$ is given on generators by

$$
d_{+1} f_{\bullet}=f_{\bullet} h_{\bullet}-l_{\bullet} f_{\bullet}, d_{+1} h_{\bullet}=g_{\bullet} f_{\bullet}-h_{\bullet} h_{\bullet}, d_{+1} g_{\bullet}=g_{\bullet} l_{\bullet}-h_{\bullet} g_{\bullet}, d_{+1} l_{\bullet}=f_{\bullet} g_{\bullet}-l_{\bullet} l_{\bullet},
$$

while $d_{-1}$ is given by

$$
d_{-1} f_{\bullet}=0, d_{-1} h_{\bullet}=-1_{\mathrm{B}}, d_{-1} g_{\bullet}=0 \text { and } d_{-1} l_{\bullet}=-1_{\mathrm{W}}
$$

We claim that

$$
\left(\mathcal{F}\left(f_{0}, f_{1}, \ldots ; g_{0}, g_{1}, \ldots\right), d_{+1}\right) \text { is acyclic in positive degrees. }
$$

We prove (12) by introducing a contracting homotopy

$$
\theta: \mathcal{F}\left(f_{0}, f_{1}, \ldots ; g_{0}, g_{1}, \ldots\right) \rightarrow \mathcal{F}\left(f_{0}, f_{1}, \ldots ; g_{0}, g_{1}, \ldots\right)
$$

as follows. Let $z_{1}, z_{2}, \ldots$ denote generators of $\mathcal{F}\left(f_{0}, f_{1}, \ldots ; g_{0}, g_{1}, \ldots\right)$, then let, for $m \geq 0$,

$$
R\left(z_{1} z_{2}\right):= \begin{cases}f_{2 m+2}, & \text { if } z_{1} z_{2}=f_{0} f_{2 m+1} \\ g_{2 m+2}, & \text { if } z_{1} z_{2}=g_{0} g_{2 m+1} \\ g_{2 m+1}, & \text { if } z_{1} z_{2}=f_{0} g_{2 m} \\ f_{2 m+1}, & \text { if } z_{1} z_{2}=g_{0} f_{2 m} \\ 0 & \text { if otherwise. }\end{cases}
$$

Then the contracting homotopy $\theta$ is defined by

$$
\theta\left(z_{1} z_{2} \cdots z_{t}\right):= \begin{cases}R\left(z_{1} z_{2}\right) z_{3} \cdots z_{t}, & \text { if } t \geq 2, \text { and } \\ 0, & \text { otherwise. }\end{cases}
$$

It is immediate to check that indeed $\theta d_{+1}(x)+d_{+1} \theta(x)=x$ whenever $x$ has positive degree, which proves (12).

Suppose that $x \in \mathcal{F}\left(f_{0}, f_{1}, \ldots ; g_{0}, g_{1}, \ldots\right)$ is a $d$-cycle of positive degree and let

$$
x=x_{1}+\cdots+x_{N}, x_{j} \text { has homogeneity } j, 1 \leq j \leq N, N>1,
$$

be its decomposition into homogeneity-homogeneous parts. Then clearly $d_{+1}\left(x_{N}\right)=$ 0 , thus, by (12), there exists some $b_{N-1}$ of homogeneity $N-1$ such that $x_{N}=$ $d_{+1}\left(b_{N-1}\right)$. Then $x-d\left(b_{N-1}\right)$ is a $d$-cycle homologous to $x$, whose decomposition contains no terms of homogeneity $\geq N$. Repeating this process as many times as necessary, we end up with some $x^{\prime}$, homologous to $x$, of homogeneity 1 , i.e. linear in the generators. An immediate inspection shows that there is no such nontrivial $x^{\prime}$ of positive degree, therefore $x^{\prime}=0$ which finishes the proof, since $x^{\prime}$ is, by construction, homologous to $x$.

Observe that, in the course of the proof of Theorem 9, we proved the following interesting statement: 
Proposition 10. The map

$$
\alpha_{+1}:\left(\mathcal{F}\left(f_{0}, f_{1}, \ldots ; g_{0}, g_{1}, \ldots\right), d_{+1}\right) \rightarrow \frac{\mathcal{F}(f, g)}{(f g=0, g f=0)}
$$

given by

$$
\alpha_{+1}\left(f_{0}\right):=[f], \alpha_{+1}\left(g_{0}\right):=[g] \text {, while } \alpha_{+1}\left(f_{n}\right)=0 \text { and } \alpha_{+1}\left(g_{n}\right)=0 \text { for } n \geq 1,
$$

is a homology isomorphism.

In the rest of this section we study when a homotopy equivalence (6) extends to a strong homotopy one. Observe first that (6) induces a 'restricted' action $A_{\text {res }}$ : $\mathcal{R}_{\text {iso }} \rightarrow \mathcal{E} n d_{M, N}$ by

$$
A_{\text {res }}\left(f_{0}\right)=F, A_{\text {res }}\left(g_{0}\right)=G, A_{\text {res }}\left(f_{1}\right)=H \text { and } A_{\text {res }}\left(g_{1}\right)=L \text {. }
$$

Related to these data are two obstruction cycles

$$
\mathfrak{o}_{M}:=F H-L F \in H_{o m}(M, N) \text { and } \mathfrak{o}_{N}:=G L-H G \in H_{o m}(N, M) .
$$

Theorem 11. The obstruction $\left[\mathfrak{o}_{M}\right] \in H_{1}(\operatorname{Hom}(M, N))$ vanishes if and only if the obstruction $\left[\mathfrak{o}_{N}\right] \in H_{1}(\operatorname{Hom}(N, M))$ does.

The restricted action $A_{\text {res }}$ can be extended to a full action $A: \mathcal{R}_{\text {iso }} \rightarrow \mathcal{E} n d_{M, N}$ if and only if one (and hence both) of the above obstructions vanish.

Proof. Let us denote by $\left(\mathcal{F}\left(f_{<n} ; g_{<n}\right), d\right)$ the suboperad of $\mathcal{F}\left(f_{0}, f_{1}, \ldots ; g_{0}, g_{1}, \ldots\right)$ generated by $\left\{f_{n}, g_{n}\right\}_{i<n}$, with the induced differential. It is clear from the definition that $d f_{n}, d g_{n} \in \mathcal{F}\left(f_{<n} ; g_{<n}\right)$ for any $n \geq 1$, thus it makes sense to consider the homology classes $\left[d f_{n}\right]_{n-1}$ and $\left[d g_{n}\right]_{n-1}$ of these elements in $H_{n-1}\left(\mathcal{F}\left(f_{<n} ; g_{<n}\right), d\right)$. We claim that

$$
\begin{aligned}
& {\left[g_{0}\right]\left[d f_{2 m}\right]_{2 m-1}+\left[d f_{2 m}\right]_{2 m-1}\left[f_{0}\right]=0 \text { in } H_{2 m-1}\left(\mathcal{F}\left(f_{<2 m} ; g_{<2 m}\right), d\right), \text { and }} \\
& {\left[f_{0}\right]\left[d f_{2 m+1}\right]_{2 m}+\left[d g_{2 m+1}\right]_{2 m}\left[f_{0}\right]=0 \text { in } H_{2 m}\left(\mathcal{F}\left(f_{<2 m+1} ; g_{<2 m+1}\right), d\right) .}
\end{aligned}
$$

The first equation follows from the inspection of the degree $2 m+1$ part of

$$
d h_{\bullet}^{2}=d\left(f_{\bullet} g_{\bullet}\right)
$$

which is

$$
\left(d h_{\bullet}^{2}\right)_{2 m+1}=f_{0}\left(d f_{2 m}\right)+\left(d g_{2 m}\right) g_{0}+d\left(\sum_{\substack{i+j=m \\ i, j \geq 1}} f_{2 i} g_{2 j}\right) ;
$$

equation (16) can be verified directly. Equation (15) follows in the same manner from

$$
d\left(f_{\bullet} h_{\bullet}-l_{\bullet} f_{\bullet}\right)=0
$$


which follows from $d^{2}=0$. Observe that (14) gives, for $m=1$,

$$
\left[g_{0}\right]\left[d f_{2}\right]_{2}+\left[d f_{2}\right]_{2}\left[f_{0}\right]=0
$$

which is mapped by $A_{\text {res }}: \mathcal{F}\left(f_{<2} ; g_{<2}\right) \rightarrow \mathcal{E} n d_{M, N}$ to

$$
[G][F H-L F]+[G L-H G][F]=0 \text { in } H_{1}(\operatorname{Hom}(M, N)),
$$

which is of course

$$
[G]\left[\mathfrak{o}_{M}\right]+\left[\mathfrak{o}_{N}\right][F]=0 .
$$

This implies the first part of the statement, since multiplication by the homology class of $f$ (resp. of $g$ ) is an isomorphism, as these maps are homotopy invertible.

Let us prove the second part of the theorem. One implication is clear - if the restricted action $A_{\text {res }}$ can be extended to a full one, then obviously both obstructions must vanish.

Suppose that both obstructions vanish. Then the restricted action can be clearly extended to $f_{2}$ and $g_{2}$, i.e. on $\mathcal{F}\left(f_{<3} ; g_{<3}\right)$; we denote this extended action by $A_{2}$.

Let us suppose that we have extended $A_{\text {res }}$ to some $A_{n-1}: \mathcal{F}\left(f_{<n} ; g_{<n}\right) \rightarrow \mathcal{E} n d_{M, N}$, $n \geq 3$, and try to extend it to $f_{n}$ and $g_{n}$. We must distinguish whether $n$ is even or odd; suppose first that $n=2 m$. The extension clearly exists if and only if

$$
\begin{array}{ll}
{\left[A_{2 m-1}\left(d f_{2 m}\right)\right]=0} & \text { in } H_{2 m-1}(\operatorname{Hom}(M, N)), \text { and } \\
{\left[A_{2 m-1}\left(d g_{2 m}\right)\right]=0} & \text { in } H_{2 m-1}(\operatorname{Hom}(N, M)) .
\end{array}
$$

This, unfortunately, need not be true in general, but we can use the following trick. Observe that if we change the definition of $A_{2 m-1}\left(f_{2 m-1}\right)$ by adding a cycle $\phi \in$ $H_{o m} m_{2 m-1}(M, M)$ and $A_{2 m-1}\left(g_{2 m-1}\right)$ by adding a cycle $\psi \in H_{o m} m_{2 m-1}(M, M)$, the extension $A_{2 m-1}$ remains well defined. We show that by such a 'recalibration,' we may always achieve that the elements in (17) vanish. Indeed, it follows from the definition of the differential, from $A_{2 m-1}\left(f_{0}\right)=F$ and $A_{2 m-1}\left(g_{0}\right)=G$, that (17) changes to

$$
[F][\phi]+\left[A_{2 m-1}\left(d f_{2 m}\right)\right]-[\psi][F]=0 \text { and }[G][\psi]+\left[A_{2 m-1}\left(d g_{2 m}\right)\right]-[\phi][G]=0 .
$$

This system can clearly be solved if and only if

$$
[G]\left[A_{2 m-1}\left(d f_{2 m}\right)\right]+\left[A_{2 m-1}\left(d g_{2 m}\right)\right][F]=0,
$$

which is the image of (14) under $A_{2 m-1}$. The case of odd $n$ is discussed in the same manner, using (15) instead of (14).

In the light of Theorem [11, we will make no distinction between $\left[\mathfrak{o}_{M}\right]$ and $\left[\mathfrak{o}_{N}\right]$ and denote both obstructions by $[\mathfrak{o}]$. The following statement is a 'chain-level' version of Theorem 11 . 
Theorem 12. The restricted action $A_{\text {res }}$ can be extended to a full action $A: \mathcal{R}_{\text {iso }} \rightarrow$ $\mathcal{E}_{n d} d_{M, N}$ by putting $A_{\text {res }}\left(f_{n}\right)=0$ and $A_{\text {res }}\left(g_{n}\right)=0$ for $n \geq 2$ if and only if the obstruction cycles (13) vanish and if $H H=0$ and $L L=0$.

The proof is an easy exercise. In [5] we formulated without proof the following theorem.

Theorem 13. Let $(F, G, H, L)$ be a homotopy equivalence (6). By changing either $H$ or $L$ we may always achieve that the obstruction $[\mathfrak{o}]$ vanishes, i.e. that, by Theorem 11, the homotopy equivalence $(F, G, H, L)$ extends to a strong one. Examples of these changes are

$$
\begin{aligned}
&(F, G, H, L) \longmapsto \\
&(F, G, H, L) \longmapsto(F, G, H-G(F H-L F), L), \text { or } \\
&(F, G, H, L-F(G L-H G)) .
\end{aligned}
$$

Proof. Let us show that the first substitution annihilates the obstructions. Denote for simplicity $H^{\prime}:=H-G(F H-L F)$. Then it can be verified directly that

$$
\begin{aligned}
& \mathfrak{o}_{M}\left(F, G, H^{\prime}, L\right)=F H-L F-F G(F H-L F)=d(-L(F H-L F)), \text { and } \\
& \mathfrak{o}_{N}\left(F, G, H^{\prime}, L\right)=G L-H G+G(F H-L F) g=d\left(H^{2} G+G L^{2}-H G L\right),
\end{aligned}
$$

therefore $\left[\mathfrak{o}_{M}\left(F, G, H^{\prime}, L\right)\right]=\left[\mathfrak{o}_{N}\left(F, G, H^{\prime}, L\right)\right]=0$. The discussion of the second substitution is the same.

\section{The retraction.}

Let us introduce a filtered colored operad $\tilde{\mathcal{R}}_{\text {iso }}$ describing perturbations of strong homotopy equivalences. It is the completion of the operad pre $\tilde{\mathcal{R}}_{\text {iso }}$ generated by two types of generators:

(i) generators $\left\{f_{n}\right\}_{n \geq 0}$ and $\left\{g_{n}\right\}_{n \geq 0}$ as in (8) for an unperturbed strongly homotopy equivalence, and

(ii) generators for a perturbation, that is, a generator $\bar{x}$ for a perturbation of the 'black' differential, a generator $\bar{y}$ for a perturbation of the 'white' differential, and generators $\bar{f}_{n}$ and $\bar{g}_{n}$ for perturbations of $f_{n}$ resp. $g_{n}, n \geq 0$.

For homogeneity of the notation, we will sometimes write $f_{n}^{0}$ (resp. $g_{n}^{0}$ ) instead of $f_{n}$ (resp. $\left.g_{n}\right)$ and $f_{n}^{1}$ (resp. $\left.g_{n}^{1}\right)$ instead of $\bar{f}_{n}$ (resp. $\bar{g}_{n}$ ). With these conventions assumed,

$$
\operatorname{pre} \tilde{\mathcal{R}}_{\text {iso }}:=\left(\mathcal{F}\left(\bar{x}, \bar{y},\left\{f_{n}^{s}\right\}_{n \geq 0}^{s=1,2},\left\{g_{n}^{s}\right\}_{n \geq 0}^{s=1,2}\right), d\right)
$$

with $\operatorname{deg}(\bar{x})=\operatorname{deg}(\bar{y})=-1$ and $\operatorname{deg}\left(f_{n}^{s}\right)=\operatorname{deg}\left(g_{n}^{s}\right)=n$. The differential $d$ will be defined later. To define on pre $\tilde{\mathcal{R}}_{\text {iso }}$ a filtration, we assign to each generator another 
degree $\underline{\text { deg }}$ by

$$
\underline{\operatorname{deg}}(\bar{x})=\underline{\operatorname{deg}}(\bar{y})=\underline{\operatorname{deg}}\left(\bar{f}_{n}\right)=\underline{\operatorname{deg}}\left(\bar{g}_{n}\right)=1, \underline{\operatorname{deg}}\left(f_{n}\right)=\underline{\operatorname{deg}}\left(g_{n}\right)=0, n \geq 0 .
$$

This assignment expresses the fact that overlined generators describe perturbations. The $\underline{d e g}$-grading of generators induces, in the standard way, a grading on pre $\tilde{\mathcal{R}}_{\text {iso }}$ and we define

$$
F^{p} \text { pre } \tilde{\mathcal{R}}_{\text {iso }}:=\left\{z \in \operatorname{pre} \tilde{\mathcal{R}}_{\text {iso }} ; \underline{\operatorname{deg}}(z) \geq p\right\}, p \geq 0 .
$$

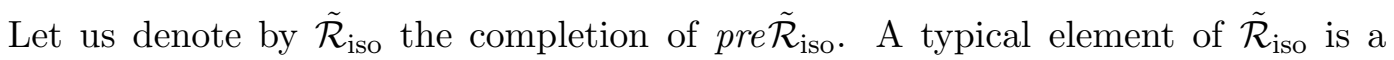
formal sum $\sum_{i \geq 0} m_{i}$ with $m_{i} \in \operatorname{pre} \tilde{\mathcal{R}}_{\text {iso }}$ and $\underline{\operatorname{deg}}\left(m_{i}\right)=i$.

The best way to describe the differential is to introduce a condensed notation (compare $(10))$ :

$$
\begin{array}{ll}
\tilde{f}_{\bullet}:=\sum_{m \geq 0} f_{2 m}+\bar{f}_{2 m}, & \tilde{g}_{\bullet}:=\sum_{m \geq 0} g_{2 m}+\bar{g}_{2 m}, \\
\tilde{h}_{\bullet}:=\sum_{m \geq 0} f_{2 m+1}+\bar{f}_{2 m+1}, & \tilde{l}_{\bullet}:=\sum_{m \geq 0} g_{2 m+1}+\bar{g}_{2 m+1} .
\end{array}
$$

The differential is given by

$$
\begin{array}{ll}
d \bar{x}=-\bar{x} \bar{x}, & d \bar{y}=-\bar{y} \bar{y}, \\
d \tilde{f}_{\bullet}=\tilde{f}_{\bullet}\left(\bar{x}+\tilde{h}_{\bullet}\right)-\left(\bar{y}+\tilde{l}_{\bullet}\right) \tilde{f}_{\bullet}, & d \tilde{g}_{\bullet}=\tilde{g}_{\bullet}\left(\bar{y}+\tilde{l}_{\bullet}\right)-\left(\bar{x}+\tilde{h}_{\bullet}\right) \tilde{g}_{\bullet}, \\
d \tilde{h}_{\bullet}=-\left(\tilde{h}_{\bullet} \bar{x}+\tilde{x}_{\bullet}\right)+\tilde{g}_{\bullet} \tilde{f}_{\bullet}-\tilde{h}_{\bullet} \tilde{h}_{\bullet}-1, & d \tilde{l}_{\bullet}=-\left(\tilde{l}_{\bullet} \bar{y}+\bar{y} \tilde{l}_{\bullet}\right)+\tilde{f}_{\bullet} \tilde{g}_{\bullet}-\tilde{l}_{\bullet} \tilde{l}_{\bullet}-1 .
\end{array}
$$

A moment's reflection shows that the differential operad $\tilde{\mathcal{R}}_{\text {iso }}$ really describes perturbations of strongly homotopy equivalences. Expanding (19) we get more explicit formulas for the differential:

$$
\begin{aligned}
d f_{2 m}^{1}:= & \sum_{t=1,2}\left(f_{2 m}^{t} \bar{x}-\bar{y} f_{2 m}^{t}\right)+\sum_{t+r \geq 1}\left(\sum_{0 \leq i<m}\left(f_{2 i}^{t} f_{2(m-i)-1}^{r}-g_{2(m-i)-1}^{t} f_{2 i}^{r}\right)\right), \\
d f_{2 m+1}^{1}:= & \sum_{t=1,2}-\left(f_{2 m+1}^{t} \bar{x}+\bar{x} f_{2 m+1}^{t}\right)+ \\
& +\sum_{t+r \geq 1}\left(\sum_{0 \leq j \leq m} g_{2 j}^{t} f_{2(m-j)}^{r}-\sum_{0 \leq j<m} f_{2 j+1}^{t} f_{2(m-j)-1}^{r}\right), \\
d g_{2 m}^{1}:= & \sum_{t=1,2}\left(g_{2 m}^{t} \bar{y}-\bar{x} g_{2 m}^{t}\right)+\sum_{t+r \geq 1}\left(\sum_{0 \leq i<m}\left(g_{2 i}^{t} g_{2(m-i)-1}^{r}-f_{2(m-i)-1}^{t} g_{2 i}^{r}\right)\right), \\
d g_{2 m+1}^{1}:= & \sum_{t=1,2}-\left(g_{2 m+1}^{t} \bar{y}+\bar{y} g_{2 m+1}^{t}\right)+ \\
& +\sum_{t+r \geq 1}\left(\sum_{0 \leq j \leq m} f_{2 j}^{t} g_{2(m-j)}^{r}-\sum_{0 \leq j<m} g_{2 j+1}^{t} g_{2(m-j)-1}^{r}\right) .
\end{aligned}
$$

The action of $d$ on $f_{n}^{0}=f_{n}$ and $g_{n}^{0}=g_{n}$ is, of course, the same as in (9). See also 8.3. The following theorem claims that $\tilde{\mathcal{R}}_{\text {iso }}$ is a resolution of the operad $\mathcal{I}$ so introduced in Example 6. 
Theorem 14. The map $\alpha: \tilde{\mathcal{R}}_{\text {iso }} \rightarrow \mathcal{I}$ so given by

$$
\alpha\left(f_{0}^{0}\right):=[f] \text { and } \alpha\left(g_{0}^{0}\right):=[g],
$$

while $\alpha$ is zero on the remaining generators, is a map of differential filtered operads that induces an isomorphism of cohomology.

Proof. It is immediate to see that $\alpha$ decomposes as $\alpha=\alpha_{\text {iso }} \circ \tilde{\alpha}$, with $\tilde{\alpha}: \tilde{\mathcal{R}}_{\text {iso }} \rightarrow \mathcal{R}_{\text {iso }}$ given by $\tilde{\alpha}\left(f_{n}^{0}\right)=f_{n}, \tilde{\alpha}\left(g_{n}^{0}\right)=g_{n}$ and $\tilde{\alpha}$ trivial on remaining generators.

Since $\alpha_{\text {iso }}$ is, by Theorem 9, a homology isomorphism, it is enough to show that $\tilde{\alpha}$ is also a homology isomorphism. This can be done by a spectral sequence argument which we omit, since we will not need the theorem in our proofs.

The philosophical meaning is that a perturbation cannot introduce nontrivial homology classes.

Let us consider the free product

$$
\mathcal{D} \text { if } * \mathcal{R}_{\text {iso }}=\left(\mathcal{F}\left(\bar{x},\left\{f_{n}\right\}_{n \geq 0},\left\{g_{n}\right\}_{n \geq 0}\right), d\right)
$$

with the differential given by (5) and (9). It is clear that the map $\iota: \mathcal{D}$ if $* \mathcal{R}_{\text {iso }} \hookrightarrow$ $\tilde{\mathcal{R}}_{\text {iso }}$ defined by

$$
\iota\left(f_{n}\right):=f_{n}^{0}, \iota\left(g_{n}\right):=g_{n}^{0} \text { and } \iota(\bar{x}):=\bar{x}, n \geq 0,
$$

is an inclusion of differential filtered colored operads. Let us formulate the main statement of this section.

Theorem 15. The operad $\mathcal{D}$ if $* \mathcal{R}_{\text {iso }}$ is a retract of $\tilde{\mathcal{R}}_{\text {iso }}$, that is, there exists a map $r: \tilde{\mathcal{R}}_{\text {iso }} \rightarrow \mathcal{D}$ if $* \mathcal{R}_{\text {iso }}$ of differential filtered colored operads such that $r \iota=\mathbb{1}_{\mathcal{D} \text { if } * \mathcal{R}_{\text {iso }}}$.

Proof. We construct the retraction $r$ explicitly. Let us define, for each odd $r \geq-1$, a 'kernel' $\mathfrak{Z}_{r}: \mathrm{B} \rightarrow \mathrm{B}, \mathfrak{Z}_{r} \in \mathcal{D}$ if $* \mathcal{R}_{\text {iso }}$, of degree $r$ by the formula

$$
\mathfrak{Z}_{r}:=\sum_{t \geq 0} \bar{x} f_{2 m_{1}+1} \bar{x} \cdots \bar{x} f_{2 m_{t}+1} \bar{x}
$$

where the summation runs over all $2\left(m_{1}+\cdots+m_{t}\right)-1=r, m_{1} \geq 0, \ldots, m_{t} \geq 0$. See 8.5 for some explicit formulas. The retraction $r: \tilde{\mathcal{R}}_{\text {iso }} \rightarrow \mathcal{D}$ if $* \mathcal{R}_{\text {iso }}$ is then given by the following formulas:

$$
\begin{aligned}
& r(\bar{x}):=\bar{x}, \\
& r(\bar{y}):=f_{0} \mathfrak{Z}_{-1} g_{0}, \\
& r\left(f_{n}\right):=f_{n}, \\
& r\left(g_{n}\right):=g_{n}, \\
& r\left(\bar{f}_{2 m}\right):=\sum_{a+b+c=m} f_{2 a} \mathfrak{Z}_{2 b-1} f_{2 c+1}, \quad r\left(\bar{g}_{2 m}\right):=\sum_{a+b+c=m} f_{2 a+1} \mathfrak{Z}_{2 b-1} g_{2 b}, \\
& r\left(\bar{f}_{2 m+1}\right):=\sum_{a+b+c=m} f_{2 a+1} \mathfrak{Z}_{2 b-1} f_{2 c+1}, \quad r\left(\bar{g}_{2 m+1}\right):=\sum_{a+b+c=m+1} f_{2 a} \mathfrak{Z}_{2 b-1} g_{2 b},
\end{aligned}
$$


where $m, n \geq 0$ and $a, b, c$ are nonnegative integers. In compact notation

$$
\mathfrak{Z}_{\bullet}:=\sum_{q \geq 0}\left(\bar{x} h_{\bullet}\right)^{q} \bar{x}
$$

we can rewrite (22) as

$$
\begin{array}{ll}
r\left(\tilde{f}_{\bullet}\right)=f_{\bullet}\left(1+\mathfrak{Z}_{\bullet} h_{\bullet}\right), & r\left(\tilde{g}_{\bullet}\right)=\left(1+h_{\bullet} \mathfrak{Z} \bullet\right) g_{\bullet}, \\
r\left(\tilde{h}_{\bullet}\right)=h_{\bullet}+h_{\bullet} \mathfrak{Z}_{\bullet} h_{\bullet}, & r\left(\bar{y}+\tilde{l}_{\bullet}\right)=l_{\bullet}+f_{\bullet} \mathfrak{Z} \bullet g_{\bullet}
\end{array}
$$

see (18) for the meaning of $\tilde{f}_{\bullet}, \tilde{g}_{\bullet}, \tilde{h}_{\bullet}$ and $\tilde{l}_{\bullet}$. It is clear that $r$ defined above is a retraction. Let us prove that it commutes with the differentials, that is

$$
d r=r d
$$

It is, of course, enough to prove $(23)$ on generators $\tilde{f}_{\bullet}, \tilde{g}_{\bullet}, \tilde{h}_{\bullet}$ and $\tilde{l}_{\bullet}$ of $\tilde{\mathcal{R}}_{\text {iso }}$. For $\tilde{f}_{\bullet}$ we have

$$
\begin{aligned}
d r\left(f_{\bullet}\right)= & d\left(f_{\bullet}\left(1+\mathfrak{Z}_{\bullet} h_{\bullet}\right)\right)=\left(f_{\bullet} h_{\bullet}-l_{\bullet} f_{\bullet}\right)\left(1+\mathfrak{Z}_{\bullet} h_{\bullet}\right)+ \\
& +f_{\bullet} \mathfrak{Z} \bullet\left(g_{\bullet} f_{\bullet}-h_{\bullet} h_{\bullet}\right) \mathfrak{Z}_{\bullet} h_{\bullet}-f_{\bullet} \mathfrak{Z} \bullet\left(g_{\bullet} f_{\bullet}-h_{\bullet} h_{\bullet}-1\right),
\end{aligned}
$$

where we used the obvious relation

$$
d \mathfrak{Z} \bullet=-\mathfrak{Z} \bullet\left(g_{\bullet} f_{\bullet}-h_{\bullet} h_{\bullet}\right) \mathfrak{Z} \bullet
$$

On the other hand,

$$
\begin{aligned}
r d\left(\tilde{f}_{\bullet}\right) & =r\left(\tilde{f}_{\bullet}\left(\bar{x}+\tilde{h}_{\bullet}\right)-\left(\bar{y}+\tilde{l}_{\bullet}\right) \tilde{f}_{\bullet}\right)= \\
& =f_{\bullet}\left(1+\mathfrak{Z}_{\bullet} h_{\bullet}\right)\left(\bar{x}+h_{\bullet}+h_{\bullet} \mathfrak{Z}_{\bullet} h_{\bullet}\right)-\left(l_{\bullet}+f_{\bullet} \mathfrak{Z}_{\bullet} g_{\bullet}\right) f_{\bullet}\left(1+\mathfrak{Z}_{\bullet} h_{\bullet}\right) .
\end{aligned}
$$

Comparing (24) to (25), using another obvious relation

$$
\bar{x}+\mathfrak{Z} \bullet h_{\bullet} \bar{x}=\mathfrak{Z} \bullet
$$

we indeed check that $r d\left(\tilde{f}_{\bullet}\right)=d r\left(\tilde{f}_{\bullet}\right)$. Equation (23) can be verified on remaining generators by the same direct argument.

\section{Proofs.}

The initial data of the Perturbation Problem define an algebra $A_{\text {in }}$ over the free product

$$
\mathcal{D} \text { if } * \mathcal{R}_{\text {fake }}=\left(\mathcal{F}\left(\bar{x}, f_{0}, g_{0}, f_{1}, g_{1}\right), d\right),
$$


of the operad $\mathcal{D}$ if of Example 7 with the operad $\mathcal{R}_{\text {fake }}$ introduced in (7), $A_{\text {in }}$ : $\mathcal{D}$ if $* \mathcal{R}_{\text {fake }} \rightarrow \mathcal{E} n d_{M, N}$, by

$A_{\text {in }}(\bar{x}):=\partial_{M}:=\tilde{d}_{M}-d_{M}, A_{\text {in }}\left(f_{0}\right):=F, A_{\text {in }}\left(g_{0}\right):=G, A_{\text {in }}\left(f_{1}\right):=H, A_{\text {in }}\left(g_{1}\right):=L$.

We seek a solution of the PP encoded to an algebra $A_{\text {out }}$ over the differential filtered suboperad

$$
\tilde{\mathcal{R}}_{\text {fake }}:=\left(\mathcal{F}\left(\bar{x}, \bar{y}, f_{0}, f_{1}, g_{0}, g_{1}, \bar{f}_{0}, \bar{f}_{1}, \bar{g}_{0}, \bar{g}_{1}\right), d\right)
$$

of the operad $\tilde{\mathcal{R}}_{\text {iso }}$ introduced in Section 5 as

$$
\begin{gathered}
\tilde{d}_{N}:=A_{\text {out }}(\bar{y}), \tilde{F}=A_{\text {out }}\left(\bar{f}_{0}\right)+F, \tilde{G}=A_{\text {out }}\left(\bar{g}_{0}\right)+G, \\
\tilde{H}:=A_{\text {out }}\left(\bar{f}_{1}\right)+H \text { and } \tilde{L}=A_{\text {out }}\left(\bar{g}_{1}\right)+L .
\end{gathered}
$$

There is a natural inclusion $\iota_{\text {fake }}: \mathcal{D}$ if $* \mathcal{R}_{\text {fake }} \hookrightarrow \tilde{\mathcal{R}}_{\text {fake }}$ given by

$$
\iota_{\text {fake }}(\bar{x}):=\bar{x}, \iota_{\text {fake }}\left(f_{0}\right):=f_{0}, \iota_{\text {fake }}\left(g_{0}\right):=g_{0}, \iota_{\text {fake }}\left(f_{1}\right):=f_{1} \text { and } \iota_{\text {fake }}\left(g_{1}\right):=g_{1} \text {. }
$$

A 'functorial' solution of the Perturbation Problem means to find a retraction

$$
r_{\text {fake }}: \tilde{\mathcal{R}}_{\text {fake }} \rightarrow \mathcal{D} \text { if } * \mathcal{R}_{\text {fake }}, r_{\text {fake }} \iota_{\text {fake }}=\mathbb{1}_{\mathcal{D} \text { if } * \mathcal{R}_{\text {fake }}} .
$$

Theorem 16. There is no retraction $r_{\text {fake }}: \tilde{\mathcal{R}}_{\text {fake }} \rightarrow \mathcal{D}$ if $* \mathcal{R}_{\text {fake }}$ as in (20).

Proof. The proof is a straightforward obstruction theory, but since the nonexistence of the retraction $r_{\text {fake }}$ motivated all this work, we reproduce the proof here in its full length. All calculations below are made modulo terms of filtration $\geq 2$, so we, in fact, work in the associated graded operad. The following equations must be satisfied (see 8.5):

$$
\begin{aligned}
d r_{\text {fake }}(\bar{y}) & =r_{\text {fake }}(d \bar{y})=0 \\
d r_{\text {fake }}\left(\bar{f}_{0}\right) & =r_{\text {fake }}\left(d \bar{f}_{0}\right)=f_{0} \bar{x}-r_{\text {fake }}(\bar{y}) f_{0} \\
d r_{\text {fake }}\left(\bar{g}_{0}\right) & =r_{\text {fake }}\left(d \bar{g}_{0}\right)=g_{0} r_{\text {fake }}(\bar{y})-\bar{x} g_{0} \\
d r_{\text {fake }}\left(\bar{f}_{1}\right) & =r_{\text {fake }}\left(d \bar{f}_{1}\right)=-\left(\bar{x} f_{1}+f_{1} \bar{x}\right)+r_{\text {fake }}\left(\bar{g}_{0}\right) f_{0}+g_{0} r_{\text {fake }}\left(\bar{g}_{0}\right), \text { and } \\
d r_{\text {fake }}\left(\bar{g}_{1}\right) & =r_{\text {fake }}\left(d \bar{g}_{1}\right)=-\left(r_{\text {fake }}(\bar{y}) g_{1}+g_{1} r_{\text {fake }}(\bar{y})\right)+r_{\text {fake }}\left(\bar{f}_{0}\right) g_{0}+f_{0} r_{\text {fake }}\left(\bar{g}_{0}\right)(30
\end{aligned}
$$

It follows from (27) and (28) that, for some $b, r_{\text {fake }}(\bar{y})=f_{0} \bar{x} g_{0}+d b$ and that

$$
r_{\text {fake }}\left(\bar{f}_{0}\right)=f_{0} \bar{x} g_{0}-b f_{0}+c_{1}, r_{\text {fake }}\left(\bar{g}_{0}\right)=f_{1} \bar{x} g_{0}+g_{0} b+c_{2},
$$

for some cycles $c_{1}, c_{2}$. The right hand side of (29) then becomes

$$
\left(g_{0} f_{0}-1\right) \bar{x} f_{1}-f_{1} \bar{x}\left(1-g_{0} f_{0}\right)+c_{2} f_{0}+g_{0} c_{1}=d\left(f_{1} \bar{x} f_{1}\right)+c_{2} f_{0}+g_{0} c_{1},
$$


while the right hand side of $(30)$ becomes

$$
\begin{array}{r}
f_{0} \bar{x}\left(f_{1} g_{0}-g_{0} g_{1}\right)+\left(f_{0} f_{1}-g_{1} f_{0}\right) \bar{x} g-b f_{0} g_{0}-d b g_{1}+f_{0} g_{0} b-g_{1} d b+c_{1} g_{0}+f_{0} c_{2}= \\
=d\left(g_{1} b-b g_{1}\right)+f_{0}\left\{\bar{x}\left(f_{1} g_{0}-g_{0} g_{1}\right)+c_{2}\right\}+\left\{\left(f_{0} f_{1}-g_{1} f_{0}\right) \bar{x}+c_{1}\right\} g_{0} .
\end{array}
$$

From this we see that (29) and (30) can be solved in $r_{\text {fake }}\left(\bar{f}_{1}\right)$ and $r_{\text {fake }}\left(\bar{g}_{1}\right)$ if and only if

$$
f_{0} \bar{x}\left(f_{1} g_{0}-g_{0} g_{1}\right)+\left(f_{0} f_{1}-g_{1} f_{0}\right) \bar{x} g_{0}
$$

is homologous to zero. It can be easily seen that this is not true.

Proof of the IPL. The initial data of the IPL can be organized into an action $E_{\text {in }}: \mathcal{D}$ if $* \mathcal{R}_{\text {iso }} \rightarrow \mathcal{E} n d_{M, N}$. Then the action

$$
E_{\text {out }}: \tilde{\mathcal{R}}_{\text {iso }} \stackrel{r}{\longrightarrow} \mathcal{D} \text { if } * \mathcal{R}_{\text {iso }} \stackrel{E_{\text {in }}}{\longrightarrow} \mathcal{E} n d_{M, N}
$$

where $r$ is the retraction of Theorem 15, clearly solves the IPL.

\section{A conceptual explanation.}

We believe in the existence of a model category (MC) structure on the category of operads. Let us ignore in this conceptual section the fact that the existence of this structure has been proved only for some special cases [3] and certainly not for the

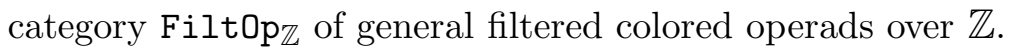

Our candidate for cofibrations in $\mathrm{FiltOp}_{\mathbb{Z}}$ are maps such that the associated maps of graded operads are cofibrations in the sense of an obvious integral version of $[5$, Definition 15] (or something close to it). Fibrations are then epimorphisms and weak equivalences are homology isomorphisms.

As argued in [5], homotopy invariant algebras are those over cofibrant operads. By Theorem 10, $\mathcal{R}_{\text {iso }}$ is a cofibrant resolution of the operad $\mathcal{I}$ so, that is why strong homotopy equivalences, as algebras over $\mathcal{R}_{\text {iso }}$, are proper homotopy versions of strict isomorphisms.

Let us show how the IPL follows from the properties of the MC structure on FiltOp $p_{\mathbb{Z}}$. The situation is summarized in the following diagram.

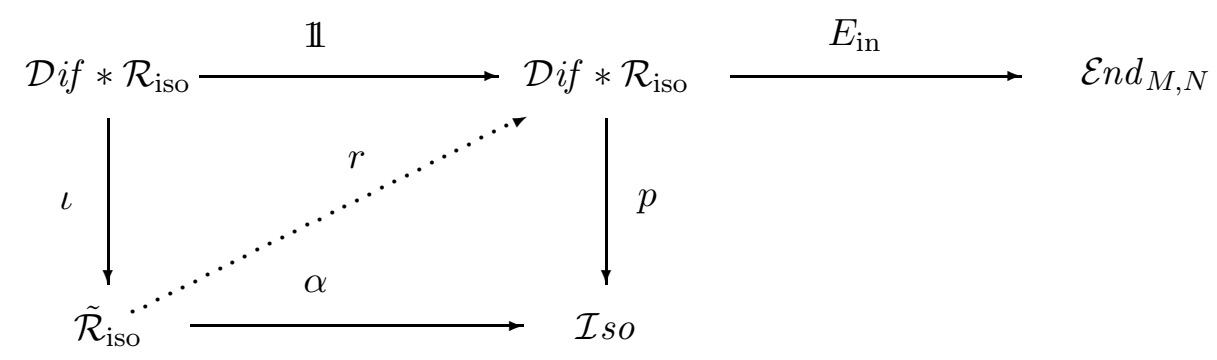


In the above diagram, $\alpha$ is the map from Theorem 14, $\iota$ the inclusion (21), $p:=\alpha \circ \iota$ and the action $E_{\text {in }}$ summarizes the input data of the IPL. The solution of the IPL will then be given by $E_{\text {out }}:=E_{\text {in }} \circ r$.

The map is $p$ clearly an epimorphism, hence a fibration. It is also a weak equivalence, because, if we ignore the acyclic (by Proposition 8) factor $\mathcal{D}$ if, the map $p$ is exactly the map $\alpha_{\text {iso }}$ of Theorem 9 . The map $\iota$ is a cofibration, thus the existence of $r$ follows from the axioms of a MC structure.

The above is, of course, just an explanation, not a proof, so we had, in the proof of Theorem 15, to construct the retraction $r$ by other means.

\section{Appendix: Explicit Formulas.}

8.1. Explicit axioms for a SHE:

$$
\begin{aligned}
d_{N} F_{0}-F_{0} d_{M} & =0 \\
d_{M} G_{0}-G_{0} d_{N} & =0 \\
d_{M} H_{1}+H_{1} d_{M} & =G_{0} F_{0}-\mathbb{1}_{M} \\
d_{N} L_{1}+L_{1} d_{N} & =F_{0} G_{0}-\mathbb{1}_{N} \\
d_{N} F_{2}-F_{2} d_{M} & =F_{0} H_{1}-L_{1} F_{0} \\
d_{M} G_{2}-G_{2} d_{N} & =G_{0} L_{1}-H_{1} G_{0} \\
d_{M} H_{3}+H_{3} d_{M} & =G_{0} F_{2}-H_{1} H_{1}+G_{2} F_{0} \\
d_{N} L_{3}+L_{3} d_{N} & =F_{0} G_{2}-L_{1} L_{1}+F_{2} G_{0} \\
d_{N} F_{4}-F_{4} d_{M} & =F_{0} H_{3}-L_{1} F_{2}+F_{2} H_{1}-L_{3} F_{0} \\
d_{M} G_{4}-G_{4} d_{N} & =G_{0} L_{3}-H_{1} G_{2}+G_{2} L_{1}-H_{3} G_{0}
\end{aligned}
$$

8.2. Formulas for the differential of $\mathcal{R}_{\text {iso }}$ :

$$
\begin{aligned}
d f_{0} & =0, & d g_{0} & =0 \\
d f_{1} & =g_{0} f_{0}-1, & d g_{1} & =f_{0} g_{0}-1, \\
d f_{2} & =f_{0} f_{1}-g_{1} f_{0}, & d g_{2} & =g_{0} g_{1}-f_{1} g_{0}, \\
d f_{3} & =g_{0} f_{2}-f_{1} f_{1}+g_{2} f_{0}, & d g_{3} & =f_{0} g_{2}-g_{1} g_{1}+f_{2} g_{0} \\
d f_{4} & =f_{0} f_{3}-g_{1} f_{2}+f_{2} f_{1}-g_{3} f_{0}, & d g_{4} & =g_{0} g_{3}-f_{1} g_{2}+g_{2} g_{1}-f_{3} g_{0},
\end{aligned}
$$


8.3. Formulas for the differential of $\tilde{\mathcal{R}}_{\text {iso }}$ : the action on $f_{n}, g_{n}$ is, for $n \geq 0$, the same as in 8.2, and

$$
\begin{aligned}
d \bar{x} & =-\bar{x} \bar{x}, d \bar{y}=-\bar{y} \bar{y}, \\
d \bar{f}_{0} & =f_{0} \bar{x}-\bar{y} f_{0}+\bar{f}_{0} \bar{x}-\bar{y} \bar{f}_{0}, \\
d \bar{g}_{0} & =g_{0} \bar{y}-\bar{x} g_{0}+\bar{g}_{0} \bar{y}-\bar{x} \bar{g}_{0}, \\
d \bar{f}_{1} & =-\left(\bar{x} f_{1}+f_{1} \bar{x}\right)+\left(\bar{g}_{0} f_{0}+g_{0} \bar{f}_{0}\right)-\left(\bar{x} \bar{f}_{1}+\bar{f}_{1} \bar{x}\right)+\bar{g}_{0} \bar{f}_{0}, \\
d \bar{g}_{1} & =-\left(\bar{y} g_{1}+g_{1} \bar{y}\right)+\left(\bar{f}_{0} g_{0}+f_{0} \bar{g}_{0}\right)-\left(\bar{y} \bar{g}_{1}+\bar{g}_{1} \bar{y}\right)+\bar{f}_{0} \bar{g}_{0}, \\
d \bar{f}_{2} & =\left(f_{2} \bar{x}-\bar{y} f_{2}\right)+\left(\bar{f}_{0} f_{1}+f_{0} \bar{f}_{1}\right)-\left(\bar{g}_{1} f_{0}+g_{1} \bar{f}_{0}\right)+\left(\bar{f}_{2} \bar{x}-\bar{y} \bar{f}_{2}\right)+\left(\bar{f}_{1} \bar{f}_{0}-\bar{g}_{1} \bar{g}_{0}\right), \\
d \bar{g}_{2} & =\left(g_{2} \bar{y}-\bar{x} g_{2}\right)+\left(\bar{g}_{0} g_{1}+g_{0} \bar{g}_{1}\right)-\left(\bar{f}_{1} g_{0}+f_{1} \bar{g}_{0}\right)+\left(\bar{g}_{2} \bar{y}-\overline{x g}_{2}\right)+\left(\bar{g}_{1} \bar{g}_{0}-\bar{f}_{1} \bar{f}_{0}\right),
\end{aligned}
$$

8.4. Formulas for the kernel $\mathfrak{Z}_{n}$ :

$$
\begin{aligned}
\mathfrak{Z}_{-1}= & \bar{x}+\bar{x} f_{1} \bar{x}+\bar{x} f_{1} \bar{x} f_{1} \bar{x}+\bar{x} f_{1} \bar{x} f_{1} \bar{x} f_{1} \bar{x}+\cdots, \\
\mathfrak{Z}_{1}= & \bar{x} f_{3} \bar{x}+\bar{x} f_{1} \bar{x} f_{3} \bar{x}+\bar{x} f_{3} \bar{x} f_{1} \bar{x}+\bar{x} f_{1} \bar{x} f_{1} \bar{x} f_{3} \bar{x}+\bar{x} f_{1} \bar{x} f_{3} \bar{x} f_{1} \bar{x}+\bar{x} f_{3} \bar{x} f_{1} \bar{x} f_{1} \bar{x}+\cdots, \\
\mathfrak{Z}_{3}= & \bar{x} f_{5} \bar{x}+\bar{x} f_{5} \bar{x} f_{1} \bar{x}+\bar{x} f_{3} \bar{x} f_{3} \bar{x}+\bar{x} f_{1} \bar{x} f_{5} \bar{x}+\bar{x} f_{1} \bar{x} f_{3} \bar{x} f_{3} \bar{x}+\bar{x} f_{3} \bar{x} f_{1} \bar{x} f_{3} \bar{x}+ \\
\quad & \quad+\bar{x} f_{3} \bar{x} f_{3} \bar{x} f_{1} \bar{x}+\bar{x} f_{1} \bar{x} f_{1} \bar{x} f_{5} \bar{x}+\bar{x} f_{1} \bar{x} f_{5} \bar{x} f_{1} \bar{x}+\bar{x} f_{5} \bar{x} f_{1} \bar{x} f_{1} \bar{x}+\cdots, \\
& \quad
\end{aligned}
$$

8.5. Formulas for the retraction $r: \tilde{\mathcal{R}}_{\text {iso }} \rightarrow \mathcal{D}$ if $* \mathcal{R}_{\text {iso }}$ :

$$
\begin{aligned}
r(\bar{y}) & :=f_{0} \mathfrak{Z}_{-1} g_{0} \\
r\left(\bar{f}_{0}\right) & :=f_{0} \mathfrak{Z}_{-1} f_{1} \\
r\left(\bar{g}_{0}\right) & :=f_{1} \mathfrak{Z}_{-1} g_{0} \\
r\left(\bar{f}_{1}\right) & :=f_{1} \mathfrak{Z}_{-1} f_{1} \\
r\left(\bar{g}_{1}\right) & :=f_{0} \mathfrak{Z}_{-1} g_{2}+f_{2} \mathfrak{Z}_{-1} g_{0}+f_{0} \mathfrak{Z}_{1} g_{0} \\
r\left(\bar{f}_{2}\right) & :=f_{0} \mathfrak{Z}_{-1} f_{3}+f_{2} \mathfrak{Z}_{-1} f_{1}+f_{0} \mathfrak{Z}_{1} f_{1} \\
r\left(\bar{g}_{2}\right) & :=f_{1} \mathfrak{Z}_{-1} g_{2}+f_{3} \mathfrak{Z}_{-1} g_{0}+f_{1} \mathfrak{Z}_{1} g_{0} \\
r\left(\bar{f}_{3}\right) & :=f_{3} \mathfrak{Z}_{-1} f_{1}+f_{1} \mathfrak{Z}_{-1} f_{3}+f_{1} \mathfrak{Z}_{1} f_{1} \\
r\left(\bar{g}_{3}\right) & :=f_{4} \mathfrak{Z}_{-1} g_{0}+f_{2} \mathfrak{Z}_{-1} g_{2}+f_{0} \mathfrak{Z}_{-1} g_{4}+f_{2} \mathfrak{Z}_{1} g_{0}+f_{0} \mathfrak{Z}_{1} g_{2}+f_{0} \mathfrak{Z}_{3} g_{0}, \\
& :
\end{aligned}
$$

8.6. Formulas for the solution of the IPL:

$$
\begin{gathered}
\tilde{d}_{N}=d_{N}+F_{0}\left(\partial_{M}+\partial_{M} H_{0} \partial_{M}+\partial_{M} H_{0} \partial_{M} H_{0} \partial_{M}+\partial_{M} H_{0} \partial_{M} H_{0} \partial_{M} H_{0} \partial_{M}+\cdots\right) G_{0}, \\
\tilde{F}_{0}=F_{0}+F_{0}\left(\partial_{M}+\partial_{M} H_{0} \partial_{M}+\partial_{M} H_{0} \partial_{M} H_{0} \partial_{M}+\partial_{M} H_{0} \partial_{M} H_{0} \partial_{M} H_{0} \partial_{M}+\cdots\right) H_{0},
\end{gathered}
$$




$$
\begin{aligned}
\tilde{G}_{0}=G_{0} & +H_{0}\left(\partial_{M}+\partial_{M} H_{0} \partial_{M}+\partial_{M} H_{0} \partial_{M} H_{0} \partial_{M}+\partial_{M} H_{0} \partial_{M} H_{0} \partial_{M} H_{0} \partial_{M}+\cdots\right) G_{0}, \\
\tilde{H}_{0}=H_{0} & +H_{0}\left(\partial_{M}+\partial_{M} H_{0} \partial_{M}+\partial_{M} H_{0} \partial_{M} H_{0} \partial_{M}+\partial_{M} H_{0} \partial_{M} H_{0} \partial_{M} H_{0} \partial_{M}+\cdots\right) H_{0}, \\
\tilde{L}_{0}=L_{0} & +F_{0}\left(\partial_{M}+\partial_{M} H_{0} \partial_{M}+\partial_{M} H_{0} \partial_{M} H_{0} \partial_{M}+\partial_{M} H_{0} \partial_{M} H_{0} \partial_{M} H_{0} \partial_{M}+\cdots\right) G_{2} \\
& +F_{2}\left(\partial_{M}+\partial_{M} H_{0} \partial_{M}+\partial_{M} H_{0} \partial_{M} H_{0} \partial_{M}+\partial_{M} H_{0} \partial_{M} H_{0} \partial_{M} H_{0} \partial_{M}+\cdots\right) G_{0} \\
& +F_{0}\left(\partial_{M} F_{3} \partial_{M}+\partial_{M} H_{0} \partial_{M} F_{3} \partial_{M}+\partial_{M} F_{3} \partial_{M} H_{0} \partial_{M}+\cdots\right) G_{0},
\end{aligned}
$$

In the above formulas, $\partial_{M}=\tilde{d}_{M}-d_{M}$.

\section{ACKNOWLEDGEMENTS}

I would like to express my thanks to Jim Stasheff and Johannes Huebschmann for reading the manuscript and many useful comments, and apologize to the latter for not implementing all his suggestions.

\section{REFERENCES}

[1] J.M. Boardman and R.M. Vogt. Homotopy Invariant Algebraic Structures on Topological Spaces. Springer-Verlag, 1973.

[2] V. Ginzburg and M.M. Kapranov. Koszul duality for operads. Duke Math. Journal, 76(1):203-272, 1994.

[3] V. Hinich. Homological algebra of homotopy algebras. Comm. in Algebra, 10(25):3291-3323, 1997.

[4] J. Huebschmann and T. Kadeishvili. Small models for chain algebras. Math. Z., 207:245-280, 1991.

[5] M. Markl. Homotopy algebras are homotopy algebras. Preprint math.AT/9907138, July 1999.

[6] J.D. Stasheff. Homotopy associativity of H-spaces I,II. Trans. Amer. Math. Soc., 108:275-312, 1963. 